\title{
Deciphering the Finger Prints of Brain Cancer Glioblastoma Multiforme from Four Different Patients by Using Near Infrared Raman Spectroscopy \\ Hirendra Nath Banerjee ${ }^{1 *}$, Arnold Banerji ${ }^{1}$, Arunendra Nath Banerjee ${ }^{1}$, Eilena Riddick ${ }^{1}$, Jenae Petis ${ }^{1}$, Shavonda Evans ${ }^{1}$, Megha Patel ${ }^{1}$, Carl Parson $^{1}$, Valerie Smith ${ }^{1}$, E.Gwebu ${ }^{1}$ and Sarah Voisin ${ }^{2}$
}

${ }^{1}$ Department of Natural Sciences and Pharmaceutical Sciences, ElizabethCity State University, University of North Carolina, ElizabethCity, NC-27909, USA ${ }^{2}$ University De Lyon, France

\begin{abstract}
To explore the effectiveness of Raman spectra to diagnose brain cancer glioblastoma multiforme (GBM), we investigated the Raman spectra of single cell from four different GBM cell lines developed from four different patients and analyzed the spectra. The Raman spectra of brain cancer (GBM) cells were similar in all these cell lines. The results indicate that Raman spectra can offer the experimental basis for the cancer diagnosis and treatment.
\end{abstract}

Keywords: Raman spectroscopy; Glioblastoma multiforme; Cancer; Optical tweezers

\section{Introduction}

Cancer is one of the most serious diseases threatening human health and life, and the influence of this disease is steadily increasing. Due to the fact that pathogeny of cancer and some correlative illnesses have not been found, and the effective diagnosis and complete therapy cannot be carried out at present, it is impossible to control the progress of the state of the illness for cancer patients in terminal stages. Therefore, early diagnosis and timely therapy is the most effective approach in improving the surviving chance of the patient. It is very difficult to diagnose cancer in modality because the early symptom of cancer is not evident and it has no distinct difference from that of some other illness. Therefore, it is a research emphasis to find new, effective diagnosis technology and treatment method [1]. At present a diagnosis of cancer is made on histological evaluation with possible prior cytological evidence, but early diagnosis of cancer affords early intervention and greatest chance of cure. Because the ability to detect early biochemical changes associated with carcinogenesis prior to the changes a pathologist identifies would revolutionize cancer diagnostics, in recent years there has been much interest in the use of optical diagnostics in cancer detection [2]. Raman spectroscopy is a non-destructive optical technique based on the inelastic scattering of monochromatic light. When a sample is irradiated, an exchange of energy takes place between the excitation light and the molecules of the sample, which results in a measurable shift in the wavelength of the incident laser light [3]. And because the energy-levels involved are unique for every molecule, the resulting Raman spectrum is essentially a 'biochemical fingerprint', containing bands representing molecular normal modes of vibration of all molecules within the interrogated region of the sample. This technique has several advantages on the other major vibrational spectroscopy technique (IR spectroscopy), which are the absence of interference due to water, sharp bands and a spatial resolution down to $\sim 1 \mu \mathrm{m}$ [4]. The oncological applications of Raman spectroscopy are already diverse and have shown to be successful in classification, discrimination, and bimolecular investigation of cancer cells [4-7]. Both Raman spectroscopy and surface enhanced Raman spectroscopy (SERS) are proving to be invaluable tools in the field of biomedical research and clinical diagnostics. The robust, compact, fit-for-purpose Raman spectrometer designs are appropriate for use in surgical procedures to help surgeons assess tumors and allow rapid decisions to be made. Raman systems are also being developed for molecular diagnostic testing to detect and measure human cancer biomarkers. Based on the SERS technique, this approach potentially could change the way bioassays are performed to improve both the sensitivity and reliability of testing. Some of the many applications of Raman and SERS in cancer biology include:

- Examination of biopsy samples

- In vitro diagnostics

- Cytology investigations at the cellular level

- Bioassay measurements

- Histopathology using microscopy

- Direct investigation of cancerous tissues

- $\quad$ Surgical targets and treatment monitoring

- Deep tissue studies

- Drug efficacy studies Astrocytes are cells found in the brain.

They are closely related to the neurons of the brain and form the blood brain barrier. Astrocytoma is Astrocyte cells that have become cancerous. These cells can form two types of tumors: The first type is benign or slow growing, and the second type is malignant or fast growing. A specific molecular diagnostic marker still does not exist for this cancer which has a very high mortality rate. We earlier reported the difference in Raman spectroscopy in normal human Astrocytes and Astrocytoma [1]. In this study we report the results of a comparison study of Raman spectroscopic analysis of Astrocytoma cell lines developed from four different male patients of different age but of same stage cancer.

*Corresponding author: Hirendra Nath Banerjee, Professor, Department of Natural Sciences, ElizabethCity State University, University of North Carolina, ElizabethCity, NC-27909, USA, Tel: 252335 3241; Fax: 252335 3629; E-mail bhirendranath@mail.ecsu.edu

Received July 29, 2014; Accepted February 10, 2015; Published February 13 2015

Citation: Banerjee HN, Banerji A, Banerjee AN, Riddick E, Petis J, et al. (2015) Deciphering the Finger Prints of Brain Cancer Glioblastoma Multiforme from Four Different Patients by Using Near Infrared Raman Spectroscopy . J Cancer Sci Ther 7: 044-047. doi:10.4172/1948-5956.1000323

Copyright: (c) 2015 Banerjee HN, et al. This is an open-access article distributed under the terms of the Creative Commons Attribution License, which permits unrestricted use, distribution, and reproduction in any medium, provided the original author and source are credited. 


\section{Materials and Methods}

\section{Cell culture}

GBM cell lines were obtained from three white male patients of different age groups HTB12,HTB14,HTB159 (obtained from ATCC,USA) and one African American male patient GBM A172 (obtained from Duke University Tisch Brain Tumor Center). HTB12, HTB14, HTB159 were cultured in L 15 medium supplemented with $10 \%$ calf serum and antibiotics in a carbon dioxide incubator at $37^{\circ} \mathrm{C}$. GBM A 172 was cultured in DMEM medium under similar conditions mentioned above. Cell viability and count was determined by the Trypan Blue method using a standard hemocytometer.

\section{Analysis of individual cells by the use of optical tweezers, and near-infrared Raman spectroscopy}

The experimental setup of the optical tweezers and Raman spectroscopy was discussed in detail previously [8]. Briefly, a laser beam at $785 \mathrm{~nm}$ from a wavelength-stabilize, beam shaped-circularized semiconductor diode laser is introduced into an inverted microscope (Nikon 2000S) through a high numerical aperture objective (100x, N. A. $=1.30$ ) to form an optical trap. The wavelength of the diode laser was temperature stabilized to avoid drifts. A cell in a liquid medium was selectively trapped with the radiation force yielded by the focused laser beam. The same laser excited Raman scattering from the trapped cell. The back scattered light was collimated with the same objective lens and passed through a 100 um confocal pinhole aperture to reject most of the off focusing Rayleigh scattering light. Two interfering notch filters were used to remove most of the onfocusing Rayleigh scattering light. The Raman scattering light was then focused onto the entrance slit of an imaging spectrograph equipped with liquid nitrogen cooled charged coupled detector (CCD). The image of the trapped cell was observed with an illumination lamp and a video camera system, the spectral resolution of the system was estimated to be $\sim 6 \mathrm{~cm}^{-1}$. In order to sample and average most of the trapped cell, the laser beam was steered rapidly $(\sim 200 \mathrm{~Hz})$ by a pair of computer controlled Galvo mirrors across the major area of the cell. Because the steering speed was very fast, the cell was found not to follow the steering beam so that the major portion of the cell was excited within the acquisition time. The acquisition time was typically 120 s for an individual cell with an excitation power of $15 \mathrm{~mW}$ at $785 \mathrm{~nm}$. Each spectrum was averaged from more than 15 separate cells and each experiment repeated three times.

\section{Software and data processing}

All our data analysis was performed with $\mathrm{R}$, using different packages (hyperSpec, MASS, permax). The raw spectra were first cut in the $500-1800 \mathrm{~cm}^{-1}$ range of the Raman shift. Then, the cut spectra were baseline corrected thanks to automatically fit polynomials: this step allows getting spectra with band edges of up to the theoretical baseline. Finally, the spectra were normalized so that the area under each curve is 1 and that the spectra are comparable. After centering the data set, a procedure of outlier removal was followed, according to the method developed by de Groot et al. [9]. After centering the data, a total of 10 PCA were performed on each of the 5 different cell lines, for the 2 sets of experiments, independently. For a given PCA, the Mahalanobis distance of each spectrum was calculated in the subspace defined by PC2 to PC8; geometrically, the Mahalanobis distance class model is an ellipsoid-shaped cluster with the population mean at its centroid and its size defined by the value of chi-squared at a user specified confidence level with $\mathrm{k}$ degrees of freedom. For example, a spectrum may be classified as a member if its Mahalanobis distance falls inside the $95 \%$ probability level. All spectra displaying a Mahalanobis distance $>18$. 48 (critical Mahalanobis distance for 7 degrees of freedom and $\mathrm{p}$-value $=0.01)$ were removed from the data set, and the procedure was repeated from the beginning until outliers were no longer detected. The use of PC scores instead of the raw data to remove the outliers eliminates collinearity problems and avoids inverting a singular matrix [10]. After the removal of outliers, the remaining spectra recorded in every given cell were averaged and all the spectra belonging to a given cell line were grouped together.

\section{Details on the parameters set for the statistical analysis}

The PMAV was applied directly to the spectral data and the Euclidean method was used to calculate pairwise distances for every permutation. After centering the data, a PCA was performed with either the 3 cell lines taken into account, using both sets of experiments ( $\mathrm{n}=8+9=17$ cells/cell line). After centering the data, several PCA were performed on couples of cell lines, using also both sets of experiments. Then, LDA, followed by the LCP, were conducted with several PCs taken into account. The automated TSPWT function in $\mathrm{R}$ uses by default the entire permutation distribution, which is only feasible if the sample sizes are fairly small. For each of our TSPWT, the sample sizes were $n=17$ cells/cell line, which is too high to use the entire distribution. Therefore, we fixed the number of permutations to $\mathrm{N}=15000$.

\section{Results}

Similarities in band intensities were observed from Astrocytoma cancer cells obtained from four different patients (Figure 1). Table 1 shows the Raman bands and their tentative molecular assignation based on earlier studies on mammalian cells $[4,7,11]$. Comparison of the band intensities in these cells showed an increase in emission at $878,1004,1264,1302,1442$ and $1660 \mathrm{~cm}$.

Our results, thus, shows distinct similarities in biochemical composition of Astrocytoma grade 4 obtained from four different patients as evidenced by band intensity in Raman spectra.

\section{Discussion}

Optical trapping is a powerful tool that uses optical forces to hold a micrometer-sized particle near the focus of a single tightly focused laser beam in three dimensions [2]. This technique has been used to capture and manipulate biological particles such as cells, bacteria, virus, and chromosomes without obvious damage using near-infrared laser beams $[3,4]$. Biomechanical properties of single cells and single biomolecules have been extensively studied with optical angular momentum of the laser beam to form micromachines $[7,11]$. The combination of Raman spectroscopy with optical trapping offers a new degree of direct analysis of chemical spectroscopic studies of single optically trapped living cells or single organelles for the purpose of the analysis and identification, without the need of mechanical immobilization or introducing biochemical stains or tags. With the introduction of vibrational spectroscopy [12-16], the constitution and conformation of macromolecules inside the trapped cells can thus be identified and studied. The essence of this paper is to demonstrate that this technique is an effective method to study in real-time changes in biochemical properties of a single cell (or organelle) which can therefore be used to detect cancer. It is known that biological cells contain a very complex mixture of organic, inorganic and biochemical components enclosed in cell membrane. A large number of biological molecules such as proteins, nucleic acids, polysaccharides, and lipids, etc. and a variety of electrolytes populate the interior of the cell. The 

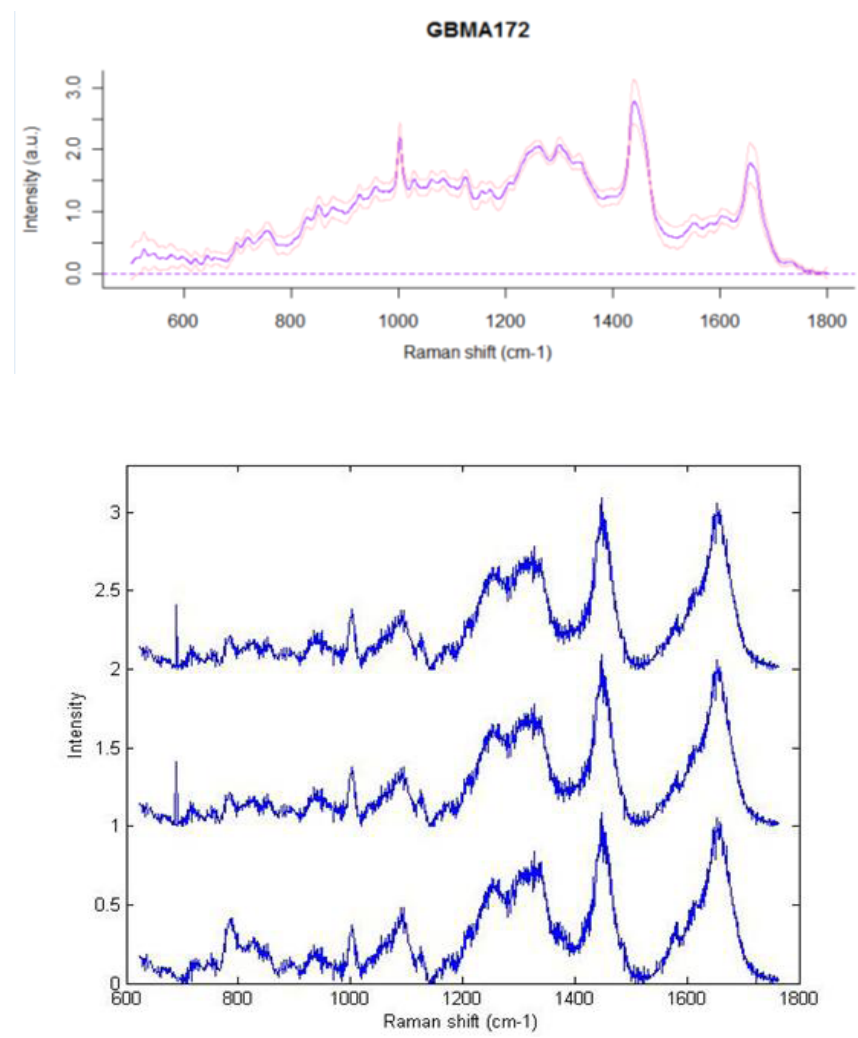

Figure 1: Raman spectra of different GBM cell lines. Each spectrum was averaged from more than 15 separate cells. The spectra of cell lines are as follows from top to bottom: GBM A172,HTB12,HTB14,HTB15.

\begin{tabular}{|c|c|}
\hline Band $\left.\mathbf{( c m}^{-1}\right)$ & Assignation \\
\hline 878 & Tyrosine(Tyr) \\
\hline 1032 & Phenylalanine \\
\hline 1302 & Amide III \\
\hline 1442 & Lipid/Protein \\
\hline 1660 & Amide I \\
\hline
\end{tabular}

Table 1: Raman bands of Astrocytoma and Astrocytes and their tentative molecula assignation.

constitution and confirmation of these biomolecules in a living cell depends on the nature of its physiological state and thus may change as a function of time in the presence of absence of external chemicals or physical agents such as temperature and/or light. It is important to notice that the nature of the biochemical properties and processes may vary from one cell species (or strain) to another. Confocal Raman spectroscopy has been applied for the analysis of chemical constituents of single living cells in different physiological stages [12-17]. The main drawback of the conventional method is that the living cells under study must be immobilize either on a glass cover slip or in a solid culture medium in order to avoid Brownian motion or cell motility when suspended in a liquid medium. Cell immobilization using a cover slip changes the chemical and physical micro environment of this living microorganism and may yield among other changes in the electrochemical potentials across the cell membrane which is known to affect cellular functions. In order to avoid this problem and consequent artifacts, we used the LTRS system that combines optical trapping and confocal Raman spectroscopy. In our scheme, optical trapping allows a mobile particle to be held at the focus of a laser beam for long-time observations and moved away from the coverslip up into solution.
Furthermore, levitations of the particle in the bathing solution causes a reduction in fluorescence and stray scattering interference form the coverslip thus undermining the weak Raman signals from the cell. In addition, optical trapping permits optimum excitation and collection for Raman scattering in a confocal configuration because the particle is maintained in the focus of the laser beam and within the focal plane of the objective. Although some degree of photo damage to the living cells may be caused by the trapping beam, this effect can be decreased substantially by using low power and selecting near-infrared laser wavelength $[9,18,19]$. Thus the vibration spectrum has promising potential as an analytical tool for diagnosing cancer because it can probe the chemical composition and molecular structure of the normal and pathological tissue, so that researchers have paid much attention to the field over the past decade. The Raman method has the relative lower requirement to the preparation of sample, thus ordinary biological sample such as cell, living tissue, DNA and RNA can be measured directly. The measurement has no damage to sample and a large number of data can easily be obtained. Therefore, many application of Raman spectrum have been gained in biology, medicine, medicament analysis and filtration $[6,7,11,20,21]$. We studied the Raman spectra of GBM cell lines obtained from three white male patients of different age groups (HTB12, HTB14, HTB15) and one African American male patient (GBM A172). GBM A172 was analyzed in a separate set up using the same technique at Purdue University while the other GBM cell lines were analyzed at University of California-Davis Biophotonics Center. The results showed Raman spectra of brain cancer cells obtained are very similar in all of these cells of same stage cancer as analyzed at the two laboratories. The results indicate that Raman spectra may offer the experimental basis for cancer diagnosis and treatment especially for brain cancer where cancer diagnosis and staging is difficult by invasive procedures. Light interacts with tissue in a number of ways including, elastic and inelastic scattering, reflection and absorption, leading to fluorescence and phosphorescence. These interactions can be used to measure abnormal changes in tissue. Initial optical biopsy systems have potential to be used as an adjunct to current investigative techniques to improve the targeting of blind biopsy. Future prospects with molecular-specific techniques may enable objective optical detection providing a real-time, highly sensitive and specific measurement of the histological state of the tissue. Raman spectroscopy has the potential to identify markers associated with malignant change and could be used as diagnostic tool for the early detection of precancerous and cancerous lesions in vivo. The clinical requirements for an objective, noninvasive, real-time probe for the accurate and repeatable measurement of pathological state of the tissue are overwhelming. Thus Raman spectroscopy will develop as a future tool for early cancer diagnostics and staging.

\section{Acknowledgement}

The authors are grateful to Dr. J. Irudyuraj of University of Purdue, IN, USA for help and Advise and Dr. Dennis Mathews at University of California-Davis Biophotonics Center. Ms. S. Evans supported by NIH-RISE grant\# R25GM082800 to Elizabeth City State University NIH-RISE program.

\section{References}

1. Banerjee H, Hawkins Z, Williams J, Blackshear M, Sawyer C, et al. (2004) Search for a novel biomarker for the brain cancer astrocytoma by using surface enhanced laser desorption/ionisation (SELDI) technique. Cell Mol Biol (Noisyle-grand) 50: 733-736.

2. Banerjee $H N$, Zhang $L$ (2007) Deciphering the finger prints of brain cancer astrocytoma in comparison to astrocytes by using near infrared Raman spectroscopy. Mol Cell Biochem 295: 237-240.

3. Diehl B, Chen CS, Grout B, Hernandez J, O'Neill S, et al. (2012) The Benefits 
Citation: Banerjee HN, Banerji A, Banerjee AN, Riddick E, Petis J, et al. (2015) Deciphering the Finger Prints of Brain Cancer Glioblastoma Multiforme from Four Different Patients by Using Near Infrared Raman Spectroscopy . J Cancer Sci Ther 7: 044-047. doi:10.4172/1948-5956.1000323

of a High-Performance, Handheld Raman Spectrometer for the Rapid Identification of Pharmaceutical Raw Materials. Eur Pharm Rev 17: 3-8.

4. Kallaway C, Almond LM, Barr H, Wood J, Hutchings J, et al. (2013) Advances in the clinical application of Raman spectroscopy for cancer diagnostics. Photodiagnosis Photodyn Ther 10: 207-219.

5. Horsnell JD, Smith JA, Sattlecker M, Sammon A, Christie-Brown J, et al. (2012) Raman spectroscopy--a potential new method for the intra-operative assessment of axillary lymph nodes. Surgeon 10: 123-127.

6. Mannie MD, Norris MS (2001) MHC class-II-restricted antigen presentation by myelin basic protein-specific CD4+ T cells causes prolonged desensitization and outgrowth of CD4- responders. Cell Immunol 212: 51-62.

7. Mannie MD, Fraser DJ, McConnell TJ (2003) IL-4 responsive CD4+ T cells specific for myelin basic protein: IL-2 confers a prolonged postactivation refractory phase. Immunol Cell Biol 81: 8-19.

8. Norris MS, McConnell TJ, Mannie MD (2001) Interleukin-2 promotes antigenic reactivity of rested $T$ cells but prolongs the postactivational refractory phase of activated T cells. Cell Immunol 211: 51-60.

9. Notingher I, Verrier S, Haque S, Polak JM, Hench LL (2003) Spectroscopic study of human lung epithelial cells (A549) in culture: living cells versus dead cells. Biopolymers 72: 230-240.

10. Otto C, Sijtsema NM, Greve J (1998) Confocal Raman microspectroscopy of the activation of single neutrophilic granulocytes. Eur Biophys J 27: 582-589.

11. Patel DM, Dudek RW, Mannie MD (2001) Intercellular exchange of class II MHC complexes: ultrastructural localization and functional presentation of adsorbed I-A/peptide complexes. Cell Immunol 214: 21-34.

12. Peticlolas WL, Patapoff TW, Thomas GA, Postlewait J, Powell JW (1996) Laser Raman microscopy of chromosomes in living eukaryotic cells: DNA polymorphism in vivo. J Raman Spectosc 27: 571.
13. Puppels GJ, de Mul FF, Otto C, Greve J, Robert-Nicoud M, et al. (1990) Studying single living cells and chromosomes by confocal Raman microspectroscopy. Nature 347: 301-333.

14. Puppels GJ, Garritsen HS, Segers-Nolten GM, de Mul FF, Greve J (1991) Raman microspectroscopic approach to the study of human granulocytes. Biophys J 60: 1046-1056.

15. Salmaso BL, Puppels GJ, Caspers PJ, Floris R, Wever R, et al. (1994) Resonance Raman microspectroscopic characterization of eosinophil peroxidase in human eosinophilic granulocytes. Biophys $\mathrm{J}$ 67: 436-446.

16. Sandstrom PA, Mannie MD, Buttke TM (1994) Inhibition of activation-induced death in T cell hybridomas by thiol antioxidants: oxidative stress as a mediator of apoptosis. J Leukoc Biol 55: 221-216.

17. Sijtsema NM, Otto C Segers-Nolten GM, Verhoeven AJ, Greve J (1998) Resonance Raman microspectroscopy of myeloperoxidase and cytochrome b558 in human neutrophilic granulocytes. Biophys J 74: 3250-3255.

18. Sijtsema NM, Tibbe AG, Segers-Nolten IG, Verhoeven AJ, Weening RS, et al. (2000) Intracellular reactions in single human granulocytes upon phorbol myristate acetate activation using confocal Raman microspectroscopy. Biophys J 78: 2606-2613.

19. Xie CA, Li YQ (2003) Confocal micro-Raman spectroscopy of single biological cells using optical trapping and shifted excitation difference techniques. J Appl Phys 93: 2982.

20. Xie C, Dinno MA, Li YQ (2002) Near-infrared Raman spectroscopy of single optically trapped biological cells. Opt Lett 27: 249-251.

21. Xie CA, Li YQ, Tang W, Newton RJ (2003) Study of dynamical process of heat denaturation in optically trapped single microorganisms by near-infrared Raman spectroscopy. J Appl Phys 94: 6138. 